\title{
Research on energy extraction characteristics of an adaptive deformation oscillating-wing
}

\author{
Bing Zhu, ${ }^{1,2}$ Wei Han, ${ }^{3}$ Xiaojing Sun, ${ }^{1,2}$ Ying Wang, ${ }^{1,2}$ Yang Cao, ${ }^{4}$ \\ Guoqing Wu, ${ }^{4}$ Diangui Huang, ${ }^{1,2, a)}$ and Zhongquan Charlie Zheng ${ }^{5}$ \\ ${ }^{1}$ Department of Energy and Power Engineering, University of Shanghai for Science \\ and Technology, Shanghai 200093, People's Republic of China \\ ${ }^{2}$ Shanghai Key Laboratory of Power Energy in Multiphase Flow and Heat Transfer, \\ Shanghai 200093, People's Republic of China \\ ${ }^{3}$ Institute of Applied Mathematics and Mechanics, Shanghai University, Shanghai 200072, \\ People's Republic of China \\ ${ }^{4}$ School of Mechanical Engineering of Nantong University, Jiangsu 226019, \\ People's Republic of China \\ ${ }^{5}$ Aerospace Engineering Department, University of Kansas, Lawrence, Kansas 66045-7621, \\ $U S A$
}

(Received 9 September 2014; accepted 20 February 2015; published online 2 March 2015)

Oscillating foil machines represent a type of flow energy harvesters which perform pitching and plunging motions simultaneously to harness the energy from incoming stream. In this paper, a new adaptive deformation oscillating wing was proposed and the theoretical performance of such a concept was studied here through unsteady two-dimensional simulations using an in-house developed computational fluid dynamics code. During operation, the proposed oscillating foil whose initial shape is symmetric can be deformed into a cambered foil, which aims to produce large lift force. Our numerical results suggest that the power efficiency of the proposed oscillating foil can be about $16.1 \%$ higher than the conventional oscillating foil without deformation. In addition, the effects of the maximum bending displacement and effective angle of attack on the efficiency of proposed oscillating foil were also discussed in this work. (C) 2015 AIP Publishing LLC.

[http://dx.doi.org/10.1063/1.4913957]

\section{NOMENCLATURE}

$b \quad$ maximum displacement of camber line from the chord line

$c \quad$ chord length of the oscillating airfoil

$C_{M} \quad$ pitching moment coefficient

$C_{X} \quad$ nondimensional coefficient of drag force in horizontal direction

$C_{Y} \quad$ nondimensional coefficient of heave force in vertical direction

$\bar{C}_{p} \quad$ average overall power coefficient

$\bar{C}_{p y} \quad$ average overall power coefficient due to heave motion

$\bar{C}_{p \theta} \quad$ average overall power coefficient due to pitch motion

$C_{p d e f}$ instantaneous power coefficient due to deformation

$d \quad$ airfoil trailing edge displacement in vertical oscillating

$f \quad$ frequency of oscillation $(=1 / T)$

$f^{*} \quad$ nondimensional frequency $\left(f^{*}=f c / U_{\infty}\right)$

$h \quad$ instantaneous vertical position of the oscillating airfoil pitch axis

$h_{0} \quad$ heaving amplitude

$l \quad$ coordinate used to specify chordwise position

$M \quad$ moment about pitching centre

a) Author to whom correspondence should be addressed. Electronic mail: dghuang@usst.edu.cn. Tel.: +86 2155897317. 
$\bar{P} \quad$ average total power extracted

$P_{y} \quad$ instantaneous total power extracted from the heave motion only

$P_{\theta} \quad$ instantaneous total power extracted from the pitch motion only

$R_{e} \quad$ Reynolds number

$S_{t} \quad$ Strouhal number $\left(S_{t}=f h_{0} / U_{\infty}\right)$

$T$ time period for one oscillation

$U_{\infty} \quad$ freestream velocity

$V_{y} \quad$ instantaneous heaving velocity

$\alpha_{0} \quad$ maximum value of the effective angle of attack $\left(\alpha_{0} \approx\left|\alpha_{T / 4}\right|\right)$

$\alpha_{T / 4} \quad$ quarter-period effective angle of attack

$\eta \quad$ efficiency of oscillating foil

$\theta \quad$ instantaneous angular position of the airfoil chord to horizontal

$\theta_{0} \quad$ pitching amplitude

$\varnothing \quad$ phase difference between pitching and heaving motion

$\omega \quad$ pitching angular velocity

\section{INTRODUCTION}

The demand for the provision of energy is increasing significantly worldwide due to population growth and economic development and will continue to grow. In the present day, over $80 \%$ of this demand is met by non-renewable, un-sustainable resources such as oil, gas, and coal. ${ }^{1}$ However, the unavoidable fact is that many fossil fuels are reaching their "peak" and are becoming depleted. Therefore, promoting exploitation of renewable energy sources appears to be one of the most efficient and effective solutions to the looming energy crisis and environmental pollution caused by the combustion of fossil fuels.

Wind and water current are two types of clean alternative energy sources. With the aid of an energy conversion device, the kinetic energy (motion) of wind or water current can be converted into mechanical energy that is used to generate electricity. Until now, various technologies for wind (water) energy conversion have been proposed. Oscillating foil machines represent a type of flow energy harvesters which extract energy from an incoming fluid flow through combined motions of plunging and pitching, as shown in Figure $1 .^{2}$ The heave-pitch oscillating airfoil motion, restricting to a pitching axis located on the chord line, can be described formally as

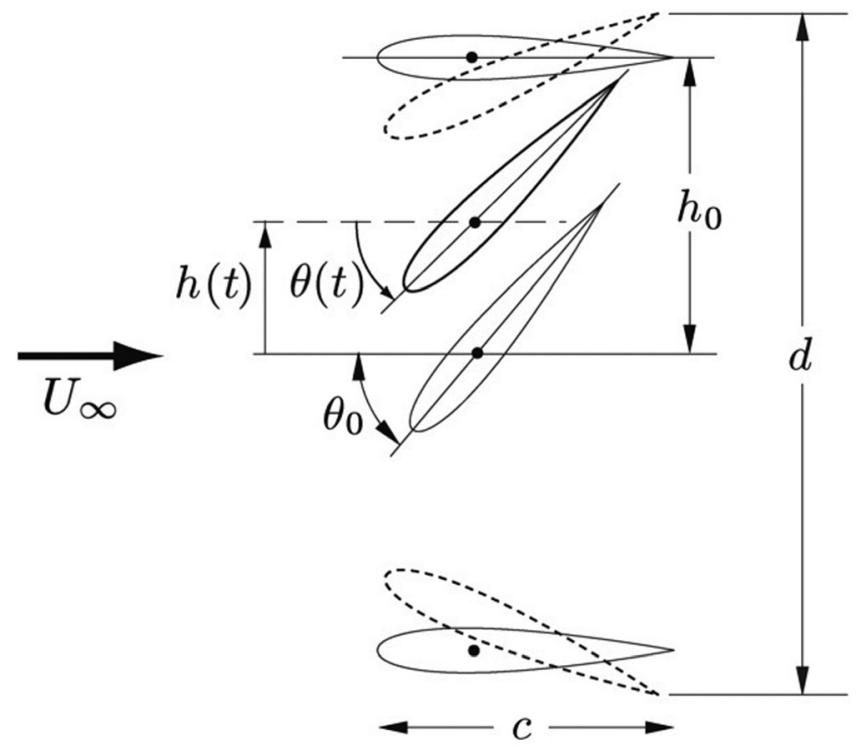

FIG. 1. Pitching and heaving motions with a phase difference of $90^{\circ} .^{2}$ 


$$
\begin{gathered}
h(t)=h_{0} \sin (2 \pi f t+\phi), \\
\theta(t)=\theta_{0} \sin (2 \pi f t),
\end{gathered}
$$

where $h_{0}$ and $\theta_{0}$ are, respectively, the heaving and pitching amplitudes, $f$ is the oscillation frequency, $\varnothing$ is the phase angle between the motions.

As there exists a plunge-induced angle of attack, the effective angle of attack which the oscillating foil experiences can be expressed as follows:

$$
\alpha(t)=\alpha_{i}(t)-\theta(t)=\arctan \left(-V_{y}(t) / U_{\infty}\right)-\theta(t)
$$

where $V_{y}$ is the heaving velocity and $U_{\infty}$ is free stream velocity. In general, the effective angle of attack reaches its maximum value at a time that is about one quarter cycle of oscillation, ${ }^{2}$

$$
\alpha_{0} \approx\left|\alpha_{T / 4}\right|=\left|\arctan \left(2 \pi f h_{0} / U_{\infty}\right)-\theta_{0}\right| .
$$

At present, for its simple geometry and application environment well-suited, there are increasing interests in the research on the kinetics and flow characteristics of oscillating foil that is used for the purpose of extracting energy from the flow of wind or water. An oscillating NACA0012 airfoil was first tested by McKinney and DeLaurier ${ }^{3}$ in a wind tunnel. The optimal value of phase angle is suggested as $90^{\circ}$. They found that when $h_{0}, \theta_{0}$, and $f^{*}$ were $0.3 c, 30^{\circ}$, and 0.12 , respectively, the efficiency of the oscillating foil was $16.8 \%$. Numerous numerical as well as experimental studies on oscillating foil turbine concepts were performed by Jones et al., ${ }^{4-6}$ and their results suggested that when $\theta_{0}<15^{\circ}$ and $f^{*}=0.25$, the maximum efficiency of oscillating foil reached $26 \%$. A parametric study of a single oscillating NACA0015 airfoil with a Reynolds number of 1100 had been analyzed by Kinsey and Dumas ${ }^{2}$ with the commercial computational fluid dynamics (CFD) code, Fluent. The airfoil underwent simple harmonic oscillating motions and the pitching point was fixed at $1 / 3$ the chord length. The results showed that the efficiency can reach as high as $34 \%$ when $0.12<f^{*}<0.18$ and $\theta_{0} \approx 70^{\circ}-80^{\circ}$. The performance of an oscillating foil is mainly governed by its motion parameters, but, in contrast, geometry parameters and viscous parameters have little effect on its efficiency. Experiments in a towing tank were conducted by Simpson ${ }^{7}$ on an oscillating NACA0012 foil with different aspect ratios. In their tests, parameters including $R_{e}=13800, h_{0} / c=1.23$, and $11^{\circ}<\theta_{0}<57^{\circ}$ were used. The experimental data indicated that when $f^{*}$ was 0.16 and the maximum angle of attack was $34.37^{\circ}$, the hydrodynamic efficiency of up to $43 \%$ was achieved. These previous studies suggested that the efficiency of the oscillating foil is still lower than that of a conventional rotary device and its performance depends severely on the settings of its motion parameters, such as the heaving amplitude, the pitching amplitude, and the frequency.

Recently, Huang and $\mathrm{Wu}^{8}$ put forward a balloon-type adaptive airfoil whose shape can be adjusted automatically based on the pressure distribution on its surface. The results showed that it considerably improves its aerodynamic characteristics under most operating conditions. To increase the efficiency of the oscillating foil, the morphing concept can be combined with the heave-pitch motions. The deformation of the foil camber-line should follow the rule to keep the motion path and the heave force in the same direction as far as possible. As seen in Figure 2, the upper surface of this airfoil bends inward forming a concave surface when it moves downward during the first half cycle; on the contrary, the upper surface deforms to become convex during the second half of cycle when it moves upward. Thus an adaptive deformation oscillating-wing aiming to obtain a potentially superior performance was proposed and will be studied in this paper.

\section{COMPUTATIONAL METHODOLOGY AND VALIDATION}

The performance of the proposed deformable oscillating airfoil was numerically studied in this paper. An in-house CFD code has been developed and used for the simulations. A detailed description of the developed code can be found in Refs. 9 and 10. 


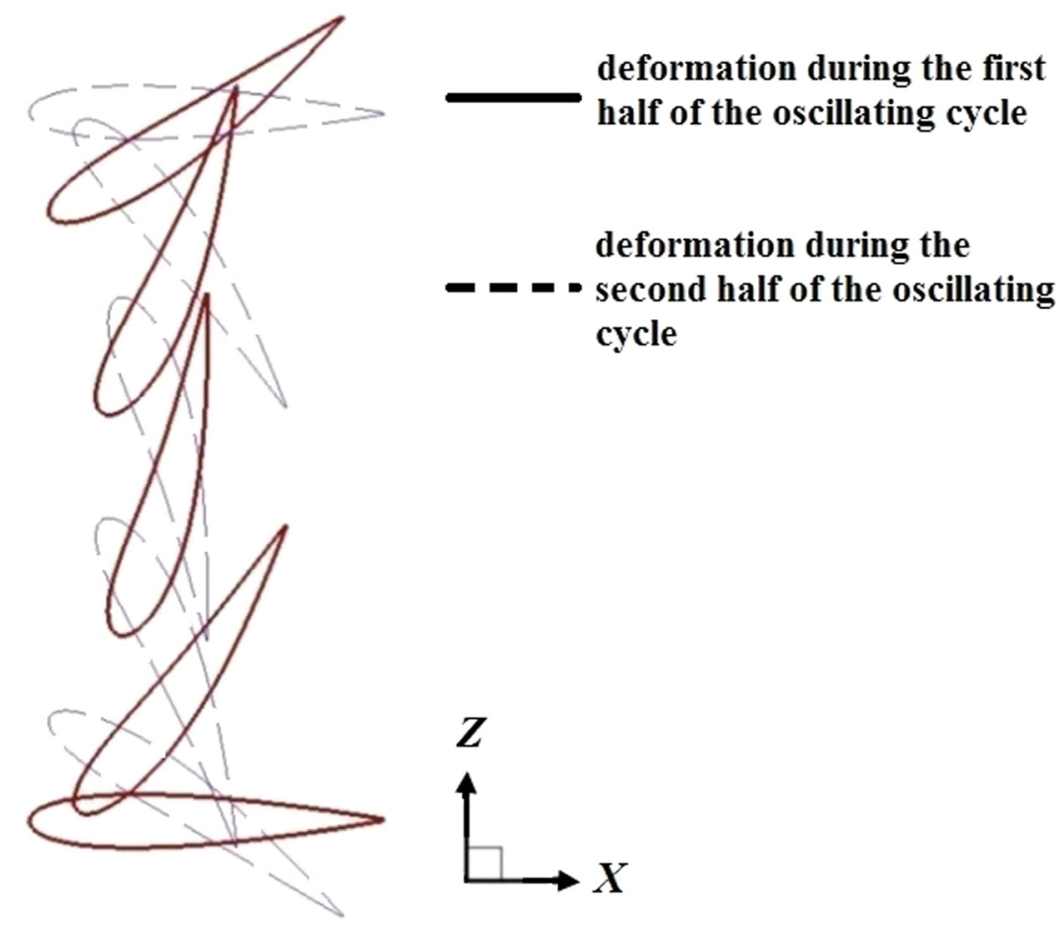

FIG. 2. Deformation of the adaptive airfoil during one cycle of oscillation.

\section{A. Governing equations}

In curvilinear coordinates, three-dimensional unsteady compressible Navier-Stokes equations can be written as follows:

$$
\frac{\partial \widehat{Q}}{\partial t}+\frac{\partial\left(\widehat{F}-\widehat{F}_{V}\right)}{\partial \xi}+\frac{\partial\left(\widehat{G}-\widehat{G}_{V}\right)}{\partial \eta}+\frac{\partial\left(\widehat{H}-\widehat{H}_{V}\right)}{\partial \zeta}=0 .
$$

The Jacobian matrix for the transformation between Cartesian and curvilinear orthogonal coordinates is in the form of the following equation:

$$
J=\frac{\partial(\xi, \eta, \zeta, t)}{\partial(x, y, z, t)} .
$$

The vector of conserved variable ( $Q$ in Cartesian coordinate) in Eq. (5) is

$$
\widehat{Q}=\frac{Q}{J}=\frac{1}{J}\left[\begin{array}{c}
\rho \\
\rho u \\
\rho v \\
\rho w \\
e
\end{array}\right] .
$$

The inviscid flux vectors are given below:

$$
\widehat{F}=\frac{F}{J}=\frac{1}{J}\left[\begin{array}{c}
\rho U \\
\rho U u+\xi_{x} p \\
\rho U v+\xi_{y} p \\
\rho U w+\xi_{z} p \\
(e+p) U-\xi_{t} p
\end{array}\right],
$$




$$
\begin{gathered}
\widehat{G}=\frac{G}{J}=\frac{1}{J}\left[\begin{array}{c}
\rho V \\
\rho V u+\eta_{x} p \\
\rho V v+\eta_{y} p \\
\rho V w+\eta_{z} p \\
(e+p) V-\eta_{t} p
\end{array}\right], \\
\widehat{H}=\frac{H}{J}=\frac{1}{J}\left[\begin{array}{c}
\rho W \\
\rho W u+\zeta_{x} p \\
\rho W v+\zeta_{y} p \\
\rho W w+\zeta_{z} p \\
(e+p) W-\zeta_{t} p
\end{array}\right] .
\end{gathered}
$$

Viscous fluxes have the following expressions:

$$
\begin{gathered}
\hat{F}_{V}=\frac{F_{V}}{J}=\frac{1}{J}\left[\begin{array}{c}
0 \\
\xi_{x} \tau_{x x}+\xi_{y} \tau_{x y}+\xi_{z} \tau_{x z} \\
\xi_{x} \tau_{x y}+\xi_{y} \tau_{y y}+\xi_{z} \tau_{y z} \\
\xi_{x} \tau_{x z}+\xi_{y} \tau_{y z}+\xi_{z} \tau_{z z} \\
\xi_{x} b_{x}+\xi_{y} b_{y}+\xi_{z} b_{z}
\end{array}\right], \\
\hat{G}_{V}=\frac{G_{V}}{J}=\frac{1}{J}\left[\begin{array}{c}
0 \\
\eta_{x} \tau_{x x}+\eta_{y} \tau_{x y}+\eta_{z} \tau_{x z} \\
\eta_{x} \tau_{x y}+\eta_{y} \tau_{y y}+\eta_{z} \tau_{y z} \\
\eta_{x} \tau_{x z}+\eta_{y} \tau_{y z}+\eta_{z} \tau_{z z} \\
\eta_{x} b_{x}+\eta_{y} b_{y}+\eta_{z} b_{z}
\end{array}\right], \\
\hat{H}_{V}=\frac{H_{V}}{J}=\frac{1}{J}\left[\begin{array}{c}
0 \\
\zeta_{x} \tau_{x x}+\zeta_{y} \tau_{x y}+\zeta_{z} \tau_{x z} \\
\zeta_{x} \tau_{x y}+\zeta_{y} \tau_{y y}+\zeta_{z} \tau_{y z} \\
\zeta_{x} \tau_{x z}+\zeta_{y} \tau_{y z}+\zeta_{z} \tau_{z z} \\
\zeta_{x} b_{x}+\eta_{y} b_{y}+\eta_{z} b_{z}
\end{array}\right] .
\end{gathered}
$$

In above equations

$$
\begin{gathered}
\tau_{x_{i} x_{j}}=\frac{M a_{\infty}}{\operatorname{Re}}\left[\mu\left(\frac{\partial u_{i}}{\partial x_{j}}+\frac{\partial u_{j}}{\partial x_{i}}\right)-\frac{2 \mu}{3} \frac{\partial u_{k}}{\partial x_{k}} \delta_{i j}\right], \\
b_{x_{i}}=u_{j} \tau_{x_{i} x_{j}}-\dot{q}_{x_{i}}, \\
\dot{q}_{x_{i}}=-\left[\frac{M a_{\infty} \mu}{\operatorname{Re} \cdot \operatorname{Pr}(\gamma-1)}\right] \frac{\partial a^{2}}{\partial x_{i}} .
\end{gathered}
$$

Contravariant velocity components are described in the following equation:

$$
\begin{aligned}
U & =\xi_{x} u+\xi_{y} v+\xi_{z} w+\xi_{t} \\
V & =\eta_{x} u+\eta_{y} v+\eta_{z} w+\eta_{t} \\
W & =\zeta_{x} u+\zeta_{y} v+\zeta_{z} w+\zeta_{t} .
\end{aligned}
$$


In addition,

$$
e=\rho H-p=\rho\left[h+\frac{1}{2}\left(u^{2}+v^{2}+w^{2}\right)\right]-p .
$$

Thus, Eq. (5) can be transformed into

$$
\frac{\partial \hat{Q}}{\partial \hat{q}} \frac{\partial \hat{q}}{\partial t}+\frac{\partial\left(\hat{F}-\hat{F}_{V}\right)}{\partial \hat{q}} \frac{\partial \hat{q}}{\partial \xi}+\frac{\partial\left(\hat{G}-\hat{G}_{V}\right)}{\partial \hat{q}} \frac{\partial \hat{q}}{\partial \eta}+\frac{\partial\left(\hat{H}-\hat{H}_{V}\right)}{\partial \hat{q}} \frac{\partial \hat{q}}{\partial \zeta}=0 .
$$

Then, velocity, pressure, and enthalpy are used as new primitive variables in the above equation,

$$
\hat{q}=\frac{q}{J}=\frac{1}{J}(p, u, v, w)^{T} .
$$

A preconditioner $\Gamma$ is then applied to replace $\frac{\partial \hat{Q}}{\partial \hat{q}}$ in Eq. (19),

$$
\begin{gathered}
\Gamma=\left[\begin{array}{ccccc}
\Theta & 0 & 0 & 0 & \rho_{h} \\
u \Theta & \rho & 0 & 0 & u \rho_{h} \\
v \Theta & 0 & \rho & 0 & v \rho_{h} \\
w \Theta & 0 & 0 & \rho & w \rho_{h} \\
H \Theta-1 & \rho u & \rho v & \rho w & H \rho_{h}+\rho
\end{array}\right], \\
\frac{\partial \hat{Q}}{\partial \hat{q}}=\left[\begin{array}{ccccc}
\rho_{p} & 0 & 0 & 0 & \rho_{h} \\
u \rho_{p} & \rho & 0 & 0 & u \rho_{h} \\
v \rho_{p} & 0 & \rho & 0 & v \rho_{h} \\
w \rho_{p} & 0 & 0 & \rho & w \rho_{h} \\
H \rho_{p}-1 & \rho u & \rho v & \rho w & H \rho_{h}+\rho
\end{array}\right] .
\end{gathered}
$$

The resulting following equation is:

$$
\Gamma \frac{\partial \hat{q}}{\partial t}+\frac{\partial\left(\hat{F}-\hat{F}_{V}\right)}{\partial \hat{q}} \frac{\partial \hat{q}}{\partial \xi}+\frac{\partial\left(\hat{G}-\hat{G}_{V}\right)}{\partial \hat{q}} \frac{\partial \hat{q}}{\partial \eta}+\frac{\partial\left(\hat{H}-\hat{H}_{V}\right)}{\partial \hat{q}} \frac{\partial \hat{q}}{\partial \zeta}=0
$$

in which

$$
\begin{gathered}
\Theta=\frac{1}{U_{\Gamma}^{2}}-\frac{\rho_{h}}{\rho}, \\
U_{\Gamma}=\left\{\begin{array}{cc}
\sqrt{\varepsilon U_{r e f}^{2}} & u^{2}+v^{2}+w^{2}<\varepsilon U_{r e f}^{2} \\
\sqrt{u^{2}+v^{2}+w^{2}} & \varepsilon U_{r e f}^{2} \leq u^{2}+v^{2}+w^{2} \leq a^{2} \\
a & u^{2}+v^{2}+w^{2}>a^{2} .
\end{array}\right.
\end{gathered}
$$

\section{B. Mesh strategy and generation}

The oscillation motion of a flexible airfoil consists of heaving, pitching, and flexible deformation. Therefore, the moving mesh method was applied to the simulation of the proposed oscillating foil. $\xi_{t}, \eta_{t}$ and $\zeta_{t}$ in Eq. (17) were not zero and their values need to be derived from the grid point velocity. For a forced oscillation of rigid airfoil (non-deformable), grid points move with the airfoil, the velocity of $x_{t}, y_{t}$, and $z_{t}$ can be directly calculated from the equations of motion of the airfoil that is provided below: 


$$
\begin{aligned}
\xi_{t} & =-\xi_{x} x_{t}-\xi_{y} y_{t}-\xi_{z} z_{t}, \\
\eta_{t} & =-\eta_{x} x_{t}-\eta_{y} y_{t}-\eta_{z} z_{t}, \\
\zeta_{t} & =-\zeta_{x} x_{t}-\zeta_{y} y_{t}-\zeta_{z} z_{t} .
\end{aligned}
$$

The proposed deformable flapping foil was then modeled by superimposing a rigid body motion and relative deformations, where the velocity of the fluid at each grid point can be worked out by superposition of these two motions.

During a mesh deformation, the computational grid cells may experience changes in their volume. Therefore, the so-called geometric conservation law that can be written in the form given in the following equation has to be satisfied:

$$
\frac{\partial}{\partial t}\left(\frac{1}{J}\right)+\frac{\partial}{\partial \xi}\left(\frac{\xi_{t}}{J}\right)+\frac{\partial}{\partial \eta}\left(\frac{\eta_{t}}{J}\right)+\frac{\partial}{\partial \zeta}\left(\frac{\zeta_{t}}{J}\right)=0,
$$

where $1 / J$ is the volume of a grid cell, $\xi_{t}, \eta_{t}$ and $\zeta_{t}$ were calculated from Eq. (26).

The rigid-deformable dynamic mesh algorithm was adopted to simulate a rigid foil performing unsteady motions (heaving and pitching). This was achieved by having the CFD code calculate the location of each node within the grid at each time step. The entire grid then can be moved together in a rigid-body motion. This ensured the spacing of the mesh remained constant throughout the entire simulation and as a result the quality of the grid setup can be maintained. ${ }^{11}$

Transfinite Interpolation (TFI) method was used for mesh deformation, which is based on the linear interpolation of the boundary motion. ${ }^{12}$ It assumes that the outer boundary condition is fixed in space while the inner boundary moves with the airfoil motion. Given the point distribution on all boundaries, this method calculates the grid points in the interior of the domain by interpolation. The motion of a node located between the moving and the fixed boundary is equal to the motion of the moving boundary times a scale factor. The advantage of using TFI is that it can generate grids conforming to specified boundaries. In the present study, the amount of deformation is small and less than 0.2 times the chord length of airfoil. Hence, the quality of the mesh produced can remain satisfactory even after a deformation has occurred. To simulate the flow around an oscillating foil, an O-type structured mesh was then used which extended about 20 chord lengths in all directions. Figures 3(a) and 3(b) show the close-up view of mesh around a deformed flexible airfoil and a rigid airfoil, respectively.

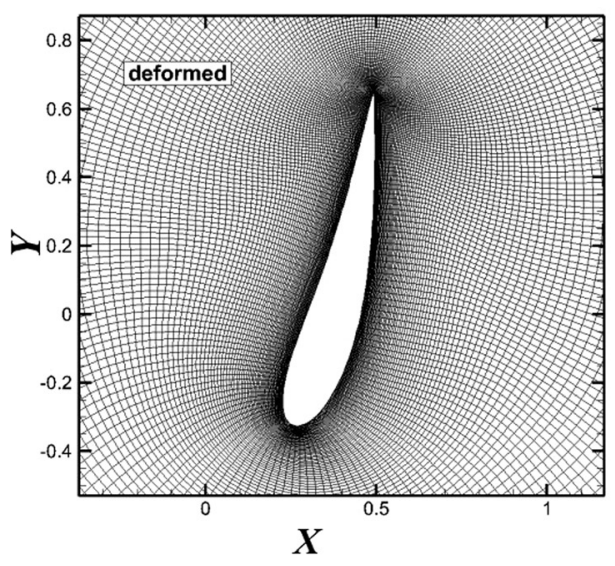

(a)

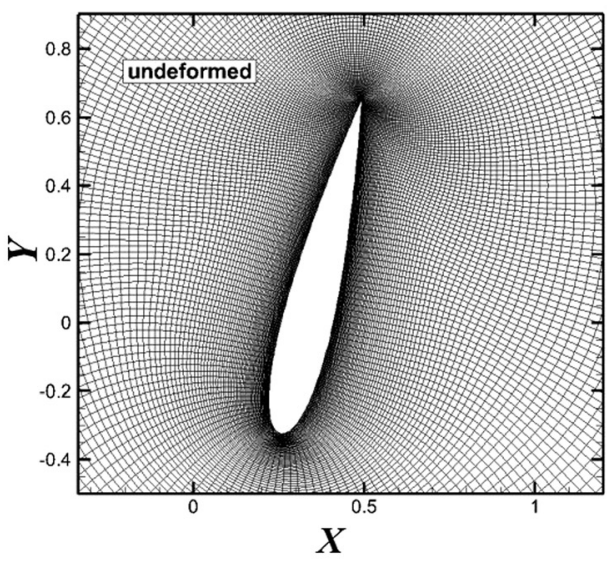

(b)

FIG. 3. The close-up view of mesh around a deformed flexible airfoil (a) and a rigid airfoil (b). 


\section{Boundary conditions and time step sizes}

A no-slip boundary condition was applied along the airfoil surface and velocity boundary condition was applied on the far-field boundary. The single equation Spalart-Allmaras (S-A) turbulence model was employed for Reynolds-Averaged Navier-Stokes (RANS) closure, due to its reasonable results for a wide range of flow problems and its simple numerical properties.

To determine the temporal resolution, the following formula was referenced at first: ${ }^{2}$

$$
\Delta t=\min \left\{\frac{T}{2000} ; \frac{c /\|V\|}{100}\right\},
$$

where $\|V\|$ is the maximum instantaneous convective flux velocity in the domain and $\mathrm{T}$ is the period of oscillation. The time step size will be examined again in Sec. IIF for the actual calculations. The numerical computation was considered converged until periodic history patterns of the monitor variables appeared; in the meantime, the difference between their cycle-averaged values from one cycle to the next should be less than $0.1 \% .^{13}$ As for the time duration needed for the simulation, it was found that when the time step size was chosen as 0.5 thousandth oscillation period $(T / 2000)$, the solution normally reached convergence over seven or eight flapping cycles.

\section{Definitions of the extracted power and efficiency} foil.

Both the pitching and heaving motions contribute to total power extracted by an oscillating

The power generated due to the heaving motion was calculated as product of heave force $Y(t)$ and heave velocity $V_{y}(t)$ as below:

$$
P_{y}(t)=Y(t) V_{y}(t) .
$$

Similarly, the extracted power from the pitching motion was calculated as product of pitching moment $M(t)$ and the angular velocity $\Omega(t)$ about the pitching pivot point

$$
P_{\theta}(t)=M_{\theta}(t) \Omega(t) .
$$

As a result, the total average power extracted over once cycle is the sum of a heaving contribution $P_{y}(t)$ and a pitching contribution $P_{\theta}(t)$ and can be computed in non-dimensional form using formula (31),

$$
\overline{C_{P}}=\overline{C_{P y}}+\overline{C_{P \theta}}=\int_{0}^{1}\left(C_{P y}+C_{P \theta}\right) d(t / T)=\int_{0}^{1}\left(C_{Y}(t) \frac{V_{y}(t)}{U_{\infty}}+C_{M}(t) \frac{\Omega(t) c}{U_{\infty}}\right) d(t / T),
$$

where $C_{Y}=\frac{Y(t)}{0.5 \rho c U_{\infty}^{2}}$ and $C_{M}=\frac{M_{\theta}(t)}{0.5 \rho c U_{\infty}^{2}}$.

Therefore, when $\bar{C}_{p}>0$, the oscillating foil harvests kinetic energy from the flow. However, when $\bar{C}_{p}<0$, it means that the oscillating foil consumes power and thus can be used for propulsion and maneuvering. The efficiency $\eta$ for the oscillating foil used as an energy extraction device was computed in this study as follows:

$$
\eta=\overline{C_{P}} \frac{c}{d} .
$$

\section{E. Rule of airfoil deformation}

The rule of the airfoil deformation over one cycle of oscillation can be described as: at the beginning of the airfoil downstroke motion, a symmetrical airfoil with no camber was chosen as initial shape at the top of the heave $(t=0 \mathrm{~s})$. The airfoil starts deforming during the 
downward movement and its trailing edge is tilted upwards. Then the deformation change effectively the airfoil from one symmetrical airfoil shape into a different cambered airfoil shape. The camber of the deformed airfoil continuously increases until it reaches the largest value at the midpoint of the heaving cycle $(t=T / 4)$. Subsequently, the camber of the deformed airfoil starts reducing and finally returns to the initial symmetrical aerofoil shape at the bottom of the heave $(t=T / 2)$. Similarly, in the second half of the oscillation, the symmetric airfoil moves upward and deforms to a cambered airfoil whose trailing edge is bent downwards. The camber of the deformed airfoil increases at first, reaches the maximum value at mid-heave plunging upward $(t=3 T / 4)$ and then decreases. At the end of one oscillation cycle, the deformed airfoil is restored to its original symmetric shape again $(t=T)$.

Therefore, the increment per unit time $\delta(l)$ of the perpendicular distance $l$ that is between a point on the airfoil surface and the chord line was defined as follows:

$$
\delta(l)=2 b \pi f \sin \left(\pi\left(l-l_{\text {leading }}\right) /\left(l_{\text {trailing }}-l_{\text {leading }}\right)\right),
$$

where $b$ is the maximum camber, namely, the maximum distance of the mean camber line from the chord line. Thus, the degree to which an airfoil is deformed can be described by this predetermined deformation parameter $b$.

At a certain moment during oscillations, the displacement of a point on the airfoil surface in the direction perpendicular to the chord line thus can be calculated with the following formula:

$$
\Delta(l, t)=\int_{0}^{t} \delta(l) \cdot \cos (2 \pi f t) \cdot d t
$$

In this study, it is assumed that the airfoil is made of perfectly elastic materials and thus can self-adaptive morph without the need for energy during one cycle of oscillation. In order to prove this assumption, a parameter called deformation power $P_{\text {def }}$ (the power required for the airfoil deformation) is defined as follows and is then calculated:

$$
P_{\text {def }}(t)=\int p V_{\text {def }} d A=\int-2 p b \pi f \cos (2 \pi f t) \sin \left(\pi\left(l-l_{\text {leading }}\right) /\left(l_{\text {trailing }}-l_{\text {leading }}\right)\right) d A .
$$

Based on $P_{d e f}$, the instantaneous power coefficient $C_{p d e f}$ can be obtained by using the following equation:

$$
C_{p d e f}=\frac{P_{d e f}}{0.5 \rho c s U_{\infty}^{3}}
$$

where $A$ is the total surface area of the foil, and $s$ is the span length of the foil.

\section{F. Sensitivity of grid and time step}

First, the unsteady flow field around a NACA0012 airfoil undergoing harmonic heaving and pitching motion for a power extraction was simulated. Parameters used for simulations were the non-dimensional heave amplitude $\left(h_{0} / c=1.23\right)$, the pitch amplitude $\left(\theta_{0}=85.82^{\circ}\right)$, and the non-dimensional flapping frequency of the foil $\left(f^{*}=0.16\right)$. The Reynolds number was 13800. A grid independence procedure was implemented over a coarse grid (8192 cells), an intermediate grid (32 768 cells), and a fine grid (131072 cells). In addition, the temporal resolution analysis was also carried out to find an appropriate time step for the present study. Three time steps $T / 500, T / 2000, T / 4000$ was examined for the fine grid. Figures 4(a)-4(c) are the variation of heave force coefficient $C_{Y}$, drag force coefficient $C_{X}$, and pitching moment coefficient $C_{M}$ over one periodic cycle, respectively. Although the trends are the same for all the variables, the distributions of the medium size grid can match more well with the fine grid than the coarse one. 


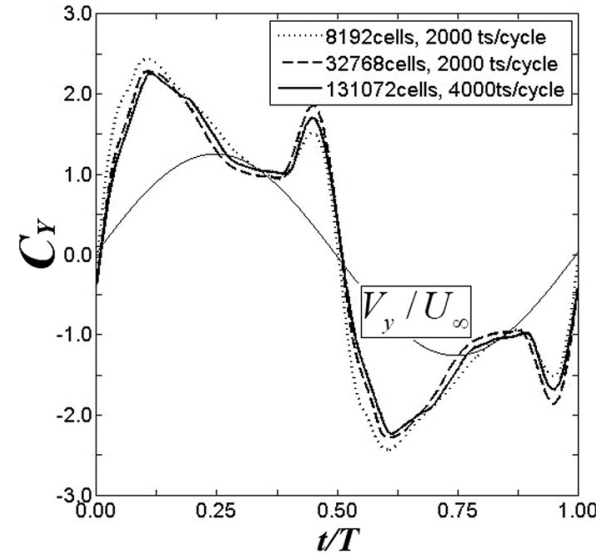

(a)

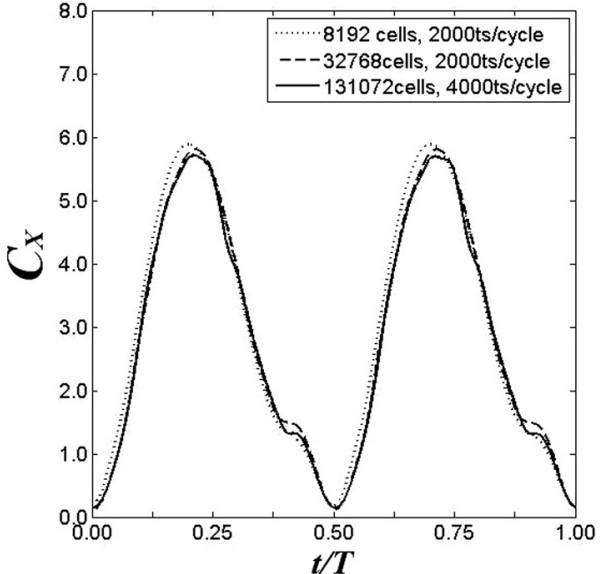

(b)

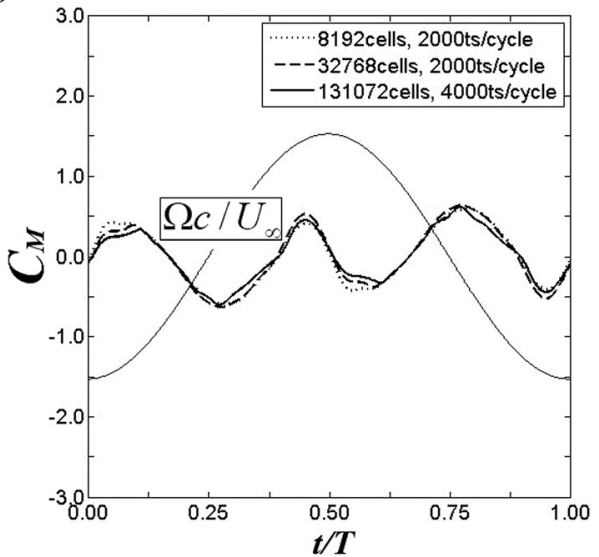

(c)

FIG. 4. Variation of $C_{X}, C_{Y}$, and $C_{M}$ along with heaving velocity over one periodic cycle.

Table I gives a summary of the average power coefficient computed on three different grids. It is noted that the value of $\bar{C}_{p_{y}}$ obtained using the medium level grids with time step size of $T / 2000$ is close to the one using the fine grid with time step size of $T / 4000$. In addition, the Heave Force Lag (HFL) (the time delay between changes in the direction of heave force and motion) obtained for these two cases are also identical. Based on the above results, the medium grid and time step size of $T / 2000$ were used for the following simulations.

\section{G. Validation}

As a means of comparison with existing established data for validation of the proposed numerical model, a NACA0012 airfoil undergoing sinusoidal oscillations was simulated in order to reproduce numerically the experimental results of Simpson et al. ${ }^{7}$ Comparison of

TABLE I. Grid and time-step sensitivity studies.

\begin{tabular}{lcccc}
\hline \hline Number of cells & $T$ s/cycle & $\bar{C}_{p_{y}}$ & $\bar{C}_{p_{\theta}}$ & HFL \\
\hline 8192 & 2000 & 1.286 & -0.094 & $0.005 T$ \\
23768 & 2000 & 1.216 & -0.059 & $0.011 T$ \\
131072 & 500 & 1.373 & -0.098 & $0.014 T$ \\
131072 & 2000 & 1.236 & -0.062 & $0.009 T$ \\
131072 & 4000 & 1.209 & -0.036 & $0.011 T$ \\
\hline \hline
\end{tabular}


experimental and numerical results is presented in Figure 5. It is observed that this computational setup yields good agreement with well validated sinusoidal motion case at the same flow conditions, lending confidence that it will provide reliable results for the following simulations. Because the real machine has a relatively high aspect ratio, the 2D approximation in simulation can give reasonable results with acceptable accuracy when we do not care about the vortex structure near the wingtip and its effect on performance.

Besides, a parametric numerical study was also conducted with the aim of identifying the optimal combination of motion parameters for the oscillating foil. Our results suggested that, in order to achieve high efficiency, the optimal motion parameters of an oscillating foil are $0.14<f^{*}<0.18,\left|\alpha_{T / 4}\right|>20^{\circ}$, and $h_{0} / c=1$. These are consistent with previously published works $^{2,14-18}$ and therefore the validity of the established numerical model was further confirmed.

Under the same operating conditions stated above, the time evolution of $C_{p d e f}$ over one heave cycle of the deformed foil is also calculated and the obtained result is displayed in Fig. 6. It can be seen that the time evolution of $C_{p d e f}$ appears to be periodic and its value is very small. The time averaged $\overline{C_{p d e f}}$ over one cycle is almost zero and therefore confirm our previous assumption in Sec. IIE that there is almost no extra power required for the foil deformation.

\section{RESULTS ANALYSIS OF AN DEFORMABLE OSCILLATION FOIL}

The 2D simulations were then conducted with the in-house developed CFD code to study the flow field about the proposed deformed oscillating airfoil (NACA0015) with $\operatorname{Re}=10000$ and the pitching point was fixed at $1 / 3$ the chord length, as illustrated in Figure 2.

\section{A. Effect of maximum camber on efficiency}

Effect of the maximum camber $b$ on the aerodynamic characteristics of the deformable oscillating foil was studied in the present work. When $b$ is a positive value, the airfoil is deformed according to the predetermined periodic law of motion described in Sec. IIE. Whereas when $b$ is a negative value, the airfoil then experiences the opposite pattern of deformation. When $b$ was $-0.063 c$ or $0.063 c$, respectively, variations of the obtained heave force with time were compared and shown in Figure 7, in which the dimensionless motion parameters

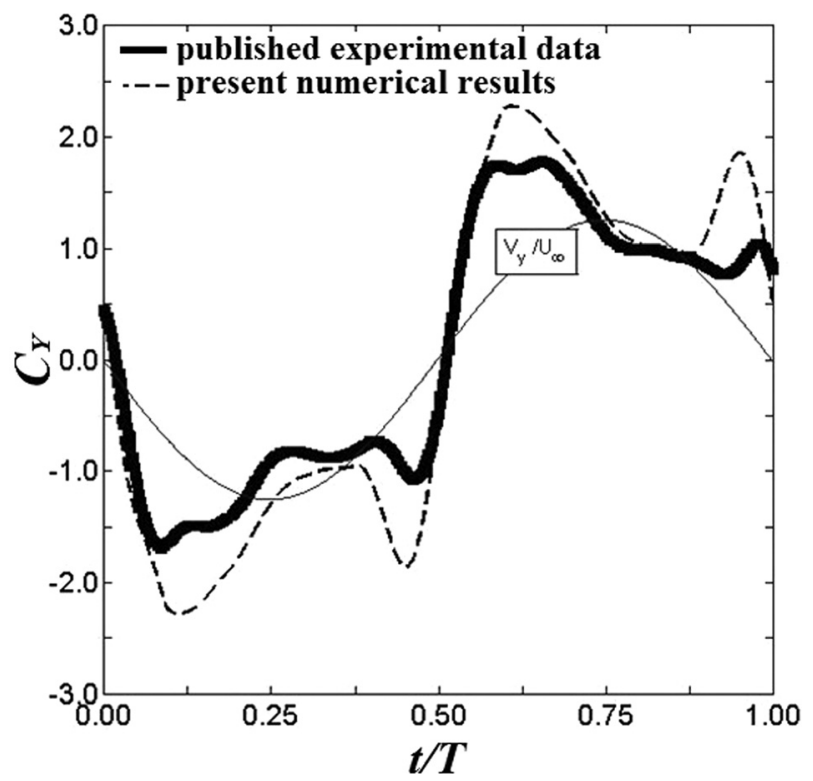

FIG. 5. Comparison of model predictions with published experimental data from Ref. 7. 


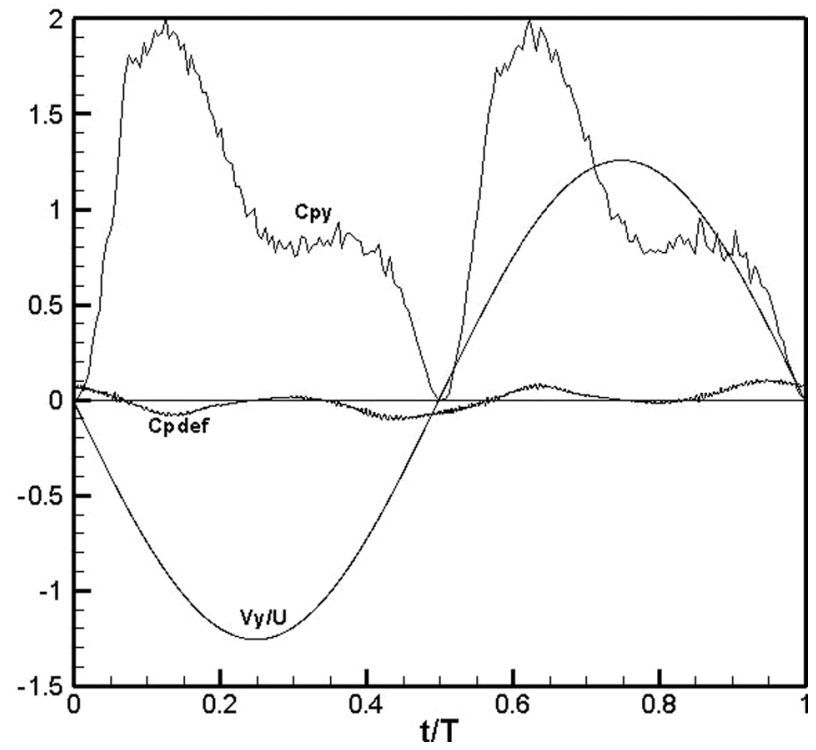

FIG. 6. The time evolution of the calculated power coefficients over one cycle of deformed foil.

of the airfoil oscillating harmonically in heave and pitch were $f^{*}=0.14, h_{0} / c=1$, and $\left|\alpha_{\mathrm{T} / 4}\right|=35^{\circ}$.

When $b$ is the positive value, the heave force is evidently higher than that of $b=0$. However, when $b$ is the negative value, the heave force is reduced compared to when $b=0$. The obtained power extraction efficiency of the deformable airfoil is summarized in Table II. It can be seen that within the given range of $b, \bar{C}_{p_{v}}$ and $\eta$ of the proposed airfoil are increased with increasing the value of $b$ and are all higher than that of the rigid airfoil $(b=0)$. When $b=0.126$, the efficiency of the deformable airfoil is the highest and is about $16.1 \%$ higher than that of the rigid airfoil.

Figures $8(\mathrm{a})$ and $8(\mathrm{~b})$ are the vorticity and pressure coefficient contours around the rigid airfoil at $t=T / 4$. When $b=-0.063$ and 0.063 , the vorticity and pressure coefficient contours

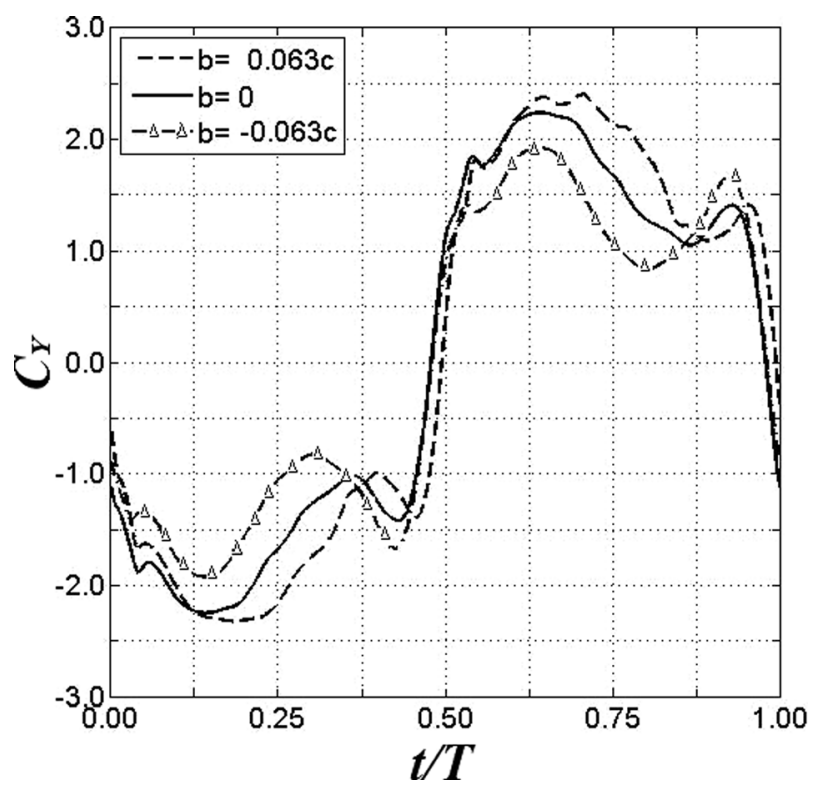

FIG. 7. Variation of heave force with time. 
TABLE II. Comparison of energy extraction between the deformable airfoil and the rigid airfoil.

\begin{tabular}{lccc}
\hline \hline $\mathrm{b}$ & $\bar{C}_{p_{y}}$ & $\bar{C}_{p_{\theta}}$ & $\eta$ \\
\hline-0.063 & 0.741 & 0.050 & 0.309 \\
0 & 0.916 & -0.011 & 0.354 \\
0.063 & 1.041 & -0.091 & 0.371 \\
0.095 & 1.073 & -0.063 & 0.395 \\
0.126 & 1.085 & -0.033 & 0.411 \\
\hline \hline
\end{tabular}
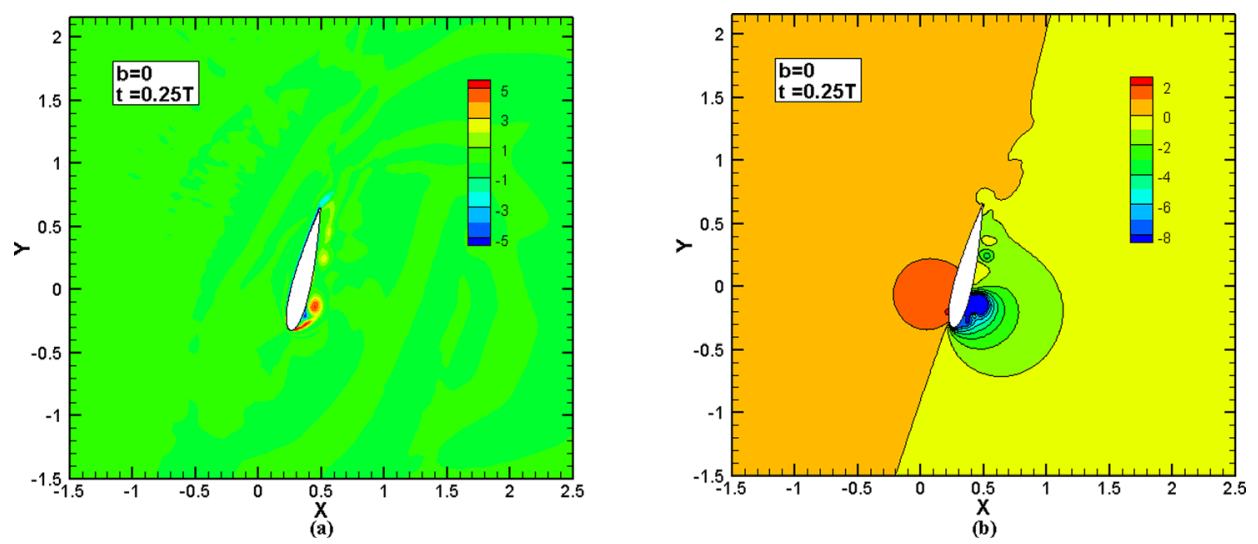

FIG. 8. Vorticity contour and pressure coefficient contour around the rigid airfoil $(b=0)$.

around the deformed airfoil in the same moment during the cycle are depicted in Figures 9(a), 9(b), 10(a), and 10(b), respectively. Compared to the rigid airfoil, the location of the leading edge vortex is quite far away from the surface of the deformed airfoil with $b$ of -0.063 and the strength of leading-edge vortex is also relatively low. Conversely, for the deformed airfoil with $b$ of 0.063 , the leading edge vortex is close to the airfoil surface and its strength is high. As a result, it can be seen from Figures 8(b), 9(b), and 10(b) that the pressure on the upper surface of the deformed airfoil with $b$ of 0.063 is apparently higher than the pressure on the upper surface of both the deformed airfoil with $b$ of -0.063 and the rigid airfoil.

\section{B. Effect of the actual angle of attack on efficiency}

In Eq. (4), when the heaving amplitude $h_{0}$ and the free stream velocity $U_{\infty}$ were kept fixed, the pitching amplitude $\theta_{0}$ was varied. The effect of actual angle of attack on efficiency of the
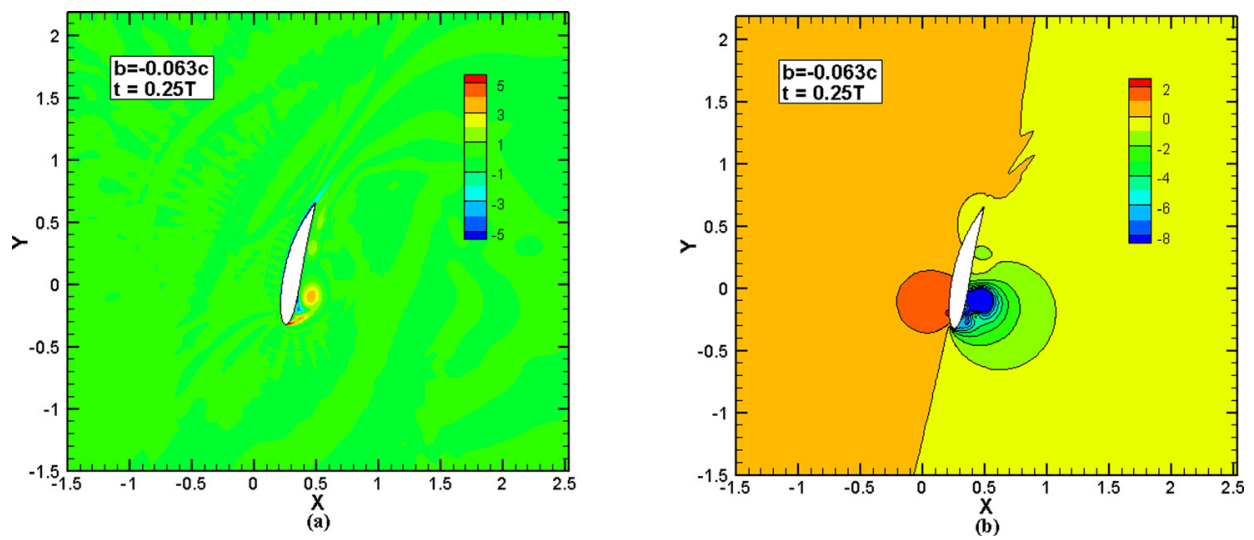

FIG. 9. Vorticity and pressure coefficient contours around the deformed airfoil $(b=-0.063 \mathrm{c})$. 

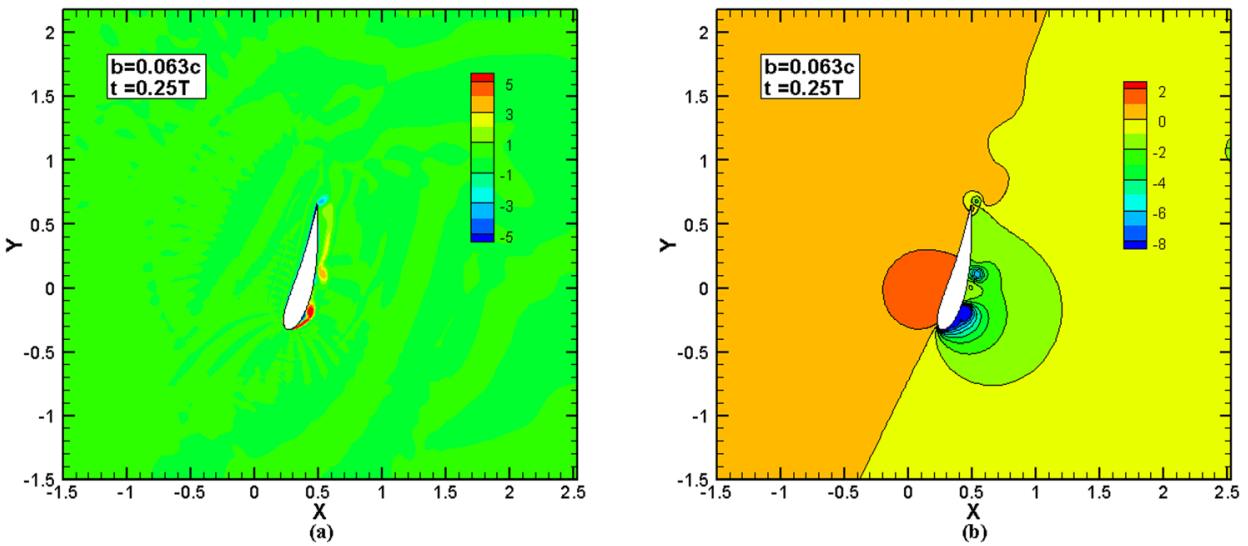

FIG. 10. Vorticity and pressure coefficient contours around the deformed airfoil $(b=0.063 \mathrm{c})$.

proposed airfoil with $b$ of 0.063 was investigated in this section. Figure 11 illustrates the heave force obtained for the rigid airfoil and the deformable airfoil when the actual angle of attack $\left|\alpha_{T / 4}\right|$ are $10^{\circ}, 20^{\circ}, 40^{\circ}$, and $50^{\circ}$, respectively.

When $\left|\alpha_{T / 4}\right|=10^{\circ}$, the heave force coefficient evolutions over one periodic cycle are quite similar for the deformable airfoil and the rigid airfoil (Figure 11(a)); when $\left|\alpha_{T / 4}\right|=20^{\circ}$, the values of the heave force coefficients of the deformable airfoil become apparently larger than those
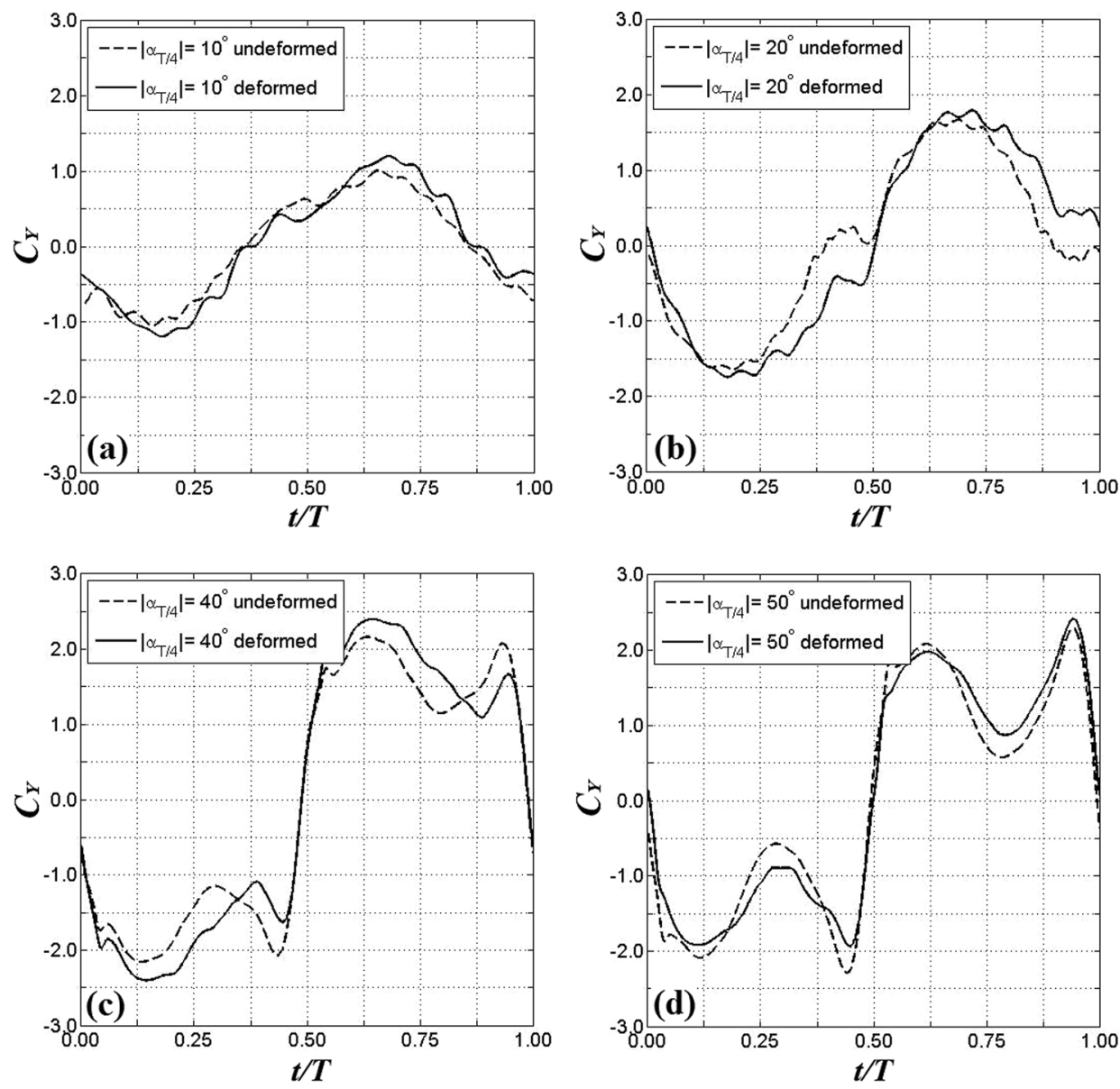

FIG. 11. Comparison of heave force coefficient between the rigid airfoil and the deformable airfoil at different $\left|\alpha_{T / 4}\right|$. 
of the rigid airfoil (Figure 11(b)); when $\left|\alpha_{T / 4}\right|=40^{\circ}$, it is found that the values of the heave force coefficients of the deformable airfoil are higher than those of the rigid airfoil during most time of one oscillation cycle (Figure 11(c)). However, when $\left|\alpha_{T / 4}\right|=50^{\circ}$, the values of the heave force coefficients of the deformable airfoil are generally less than those of the rigid airfoil despite high heave force coefficient occurred around $T / 4$ and $3 T / 4$ (Figure 11(d)).

Figure 12 shows a comparison of the efficiency of proposed airfoil and rigid airfoil at different $\left|\alpha_{T / 4}\right|$. The efficiency of the deformable airfoil can be $30 \%$ higher than that of the rigid airfoil when $\left|\alpha_{T / 4}\right|=20^{\circ}$. This improvement in efficiency is less marked in the range of $30^{\circ}$ and $40^{\circ}$. However, once $\left|\alpha_{T / 4}\right|$ rises beyond $50^{\circ}$, the efficiency of the proposed airfoil appears to be less than that of the rigid airfoil.

\section{Performance of the deformable airfoil having low thickness}

Previous studies by our group showed that the thin oscillating airfoil has a negative contribution to the heave force and power extraction. But the performance of the deformable airfoil having low thickness is unknown. Here, the energy extraction performance of a thin airfoil whose camber could be smoothly varied from zero to the desired extent was studied. The thin airfoil used for simulations was NACA0002 and the key parameters used for simulation were $b=0.063 c, \operatorname{Re}=13800, f^{*}=0.16, h_{0} / c=1.23,\left|\alpha_{T / 4}\right|=34.4^{\circ}$ (the corresponding pitching amplitude $\theta_{0}=85.82^{\circ}$ ), and the pitching axis was at one-third of the chord length from the leading edge.

A comparison of the heave force on the airfoil for the deformed NACA0002 and the rigid one is presented in Figure 13. It is found that the heave force on the deformed airfoil is almost twice that of the rigid airfoil at around $t=T / 4$ and $3 T / 4$. The resulting mean power coefficient of the deformed airfoil is 1.128 and is about $15.7 \%$ higher than the original rigid airfoil.

Figures 14 and 15 are the vorticity contour around the rigid and deformed NACA0002 airfoil at two instants in the cycle, respectively. When $t=0.125 T$, it is noted that the vortex shedding from the leading edge of the deformed airfoil is delayed compared to the rigid airfoil. Moreover, when $t=0.25 T$, the leading edge vortex shed from the deformed airfoil can still maintain high strength and stay close to the surface of airfoil. Therefore, the camber-wise deformation of the airfoil can affect the timing of the leading edge vortex shedding, the distance

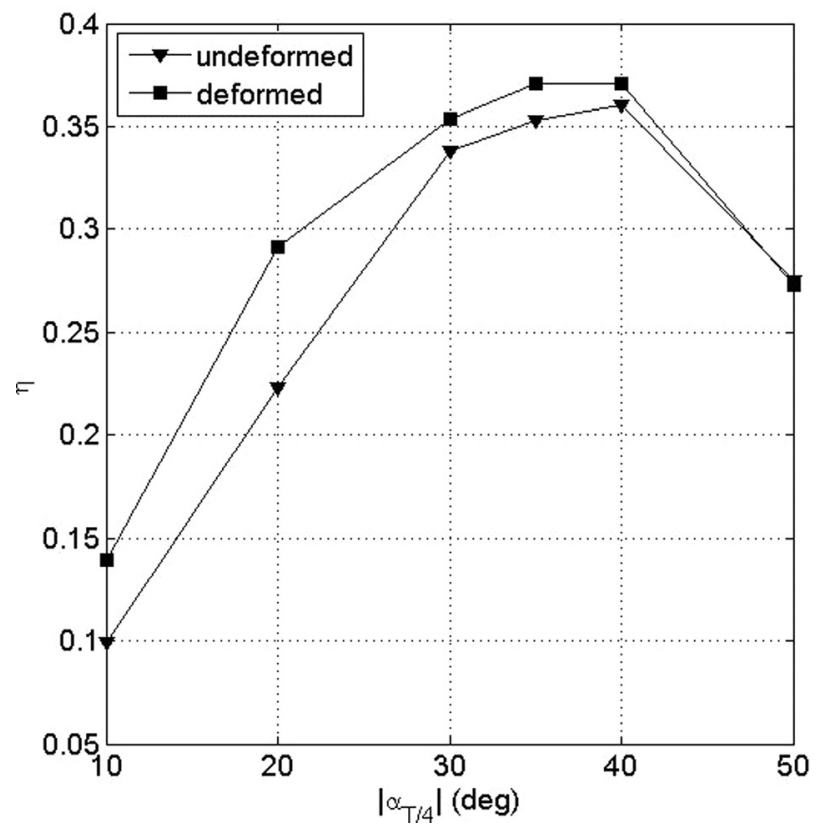

FIG. 12. Comparison of efficiency of the deformed airfoil and the rigid airfoil at different $\left|\alpha_{T / 4}\right|$. 


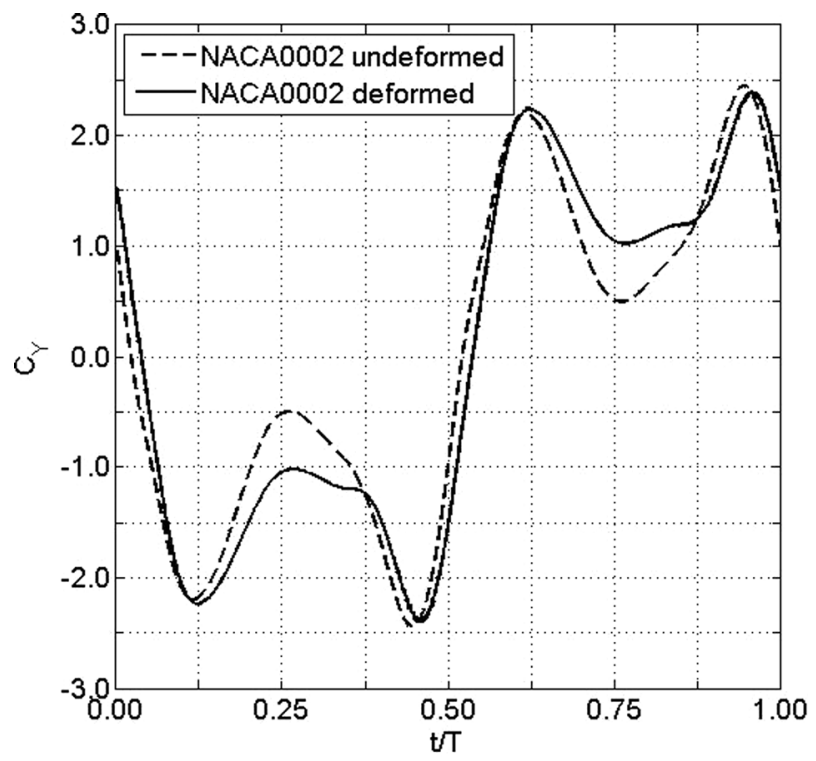

FIG. 13. Instantaneous heave force coefficient for rigid and deformable NACA0002 airfoils.
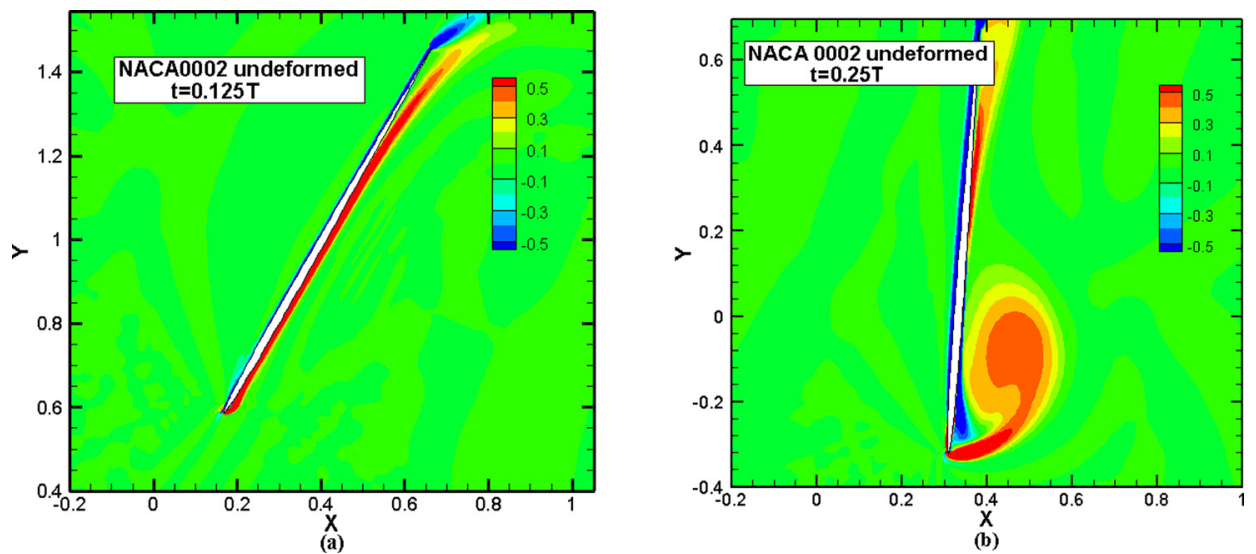

FIG. 14. Contours of vorticity around the rigid airfoil at two instants in the cycle.
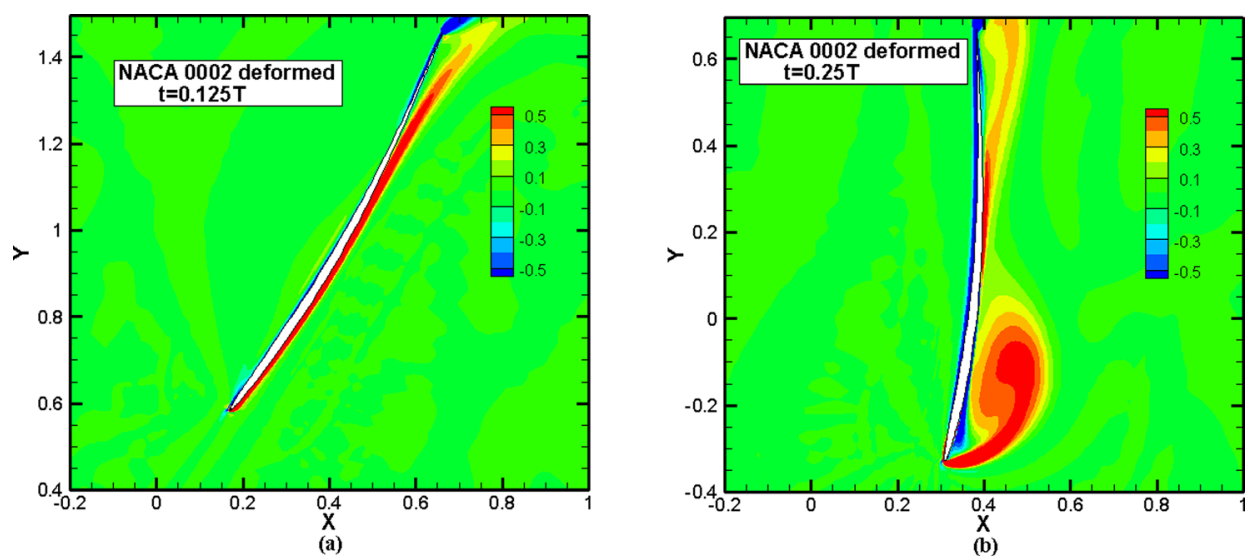

FIG. 15. Contours of vorticity around the deformed airfoil at two instants in the cycle. 
of the shed vortex from the airfoil and the strengthen of the vortex. When the value of the deformation parameter $b$ is positive, the deformable airfoil can thus hold the promise of significant performance enhancements over rigid airfoil due to the flexible deformation of their cambered shape responding to incoming airflows.

\section{CONCLUSIONS}

In this paper, the energy extraction performance of an oscillating deformable airfoil is studied numerically using the in-house developed CFD code. Based on the above results and discussion, the following conclusions were deduced:

(1) It is shown that, for proper motion and deformation parameter values, the proposed adaptive deformation oscillating-wing exhibits a superior energy extraction performance than the conventional oscillating foil.

(2) In the course of harvesting energy in uniform flows, the efficiency of the deformable airfoil will be considerably influenced by the values of $b$ which determines the deformed shape of airfoil. When the deformation parameter $b$ is a positive value, the oscillating deformable airfoil can achieve higher efficiency than the conventional oscillating airfoil. Conversely, when $b$ is a negative value, the efficiency of the deformed airfoil becomes lower than that of the rigid airfoil.

(3) There should be an optimal value of $\left|\alpha_{T / 4}\right|$ that increases the efficiency of the deformable airfoil to the maximum extent.

(4) The energy extraction efficiency of the thin airfoil can also be improved by using a deformable airfoil.

\section{ACKNOWLEDGMENTS}

This work was supported by National Natural Science Foundation of China Grant (Grant Nos. 50836006 and 11202123).

${ }^{1}$ K. Kaygusuz, "Hydropower and the world's energy future," Energy Sources 26(3), 215-224 (2004).

${ }^{2}$ T. Kinsey and G. Dumas, "Parametric study of an oscillating airfoil in a power-extraction regime," AIAA J. 46(6), 1318-1330 (2008).

${ }^{3}$ W. McKinney and J. DeLaurier, "Windmill: An oscillating-wing windmill," J. Energy 5(2), 109-115 (1981).

${ }^{4}$ K. D. Jones and M. F. Platzer, "Numerical computation of flapping-wing propulsion and power extraction," in 35th Aerospace Sciences Meeting and Exhibit, January 1997, Paper No. AIAA-97-0826, pp.1-16.

${ }^{5}$ K. D. Jones, S. Davids, and M. F. Platzer, "Oscillating-wing power generator," in ASME/JSME Joint Fluids Engineering Conference, San Francisco, California, 18-23 July 1999.

${ }^{6}$ K. D. Jones, K. Lindsey, and M. F. Platzer, "An investigation of the fluid-structure interaction in an oscillating-wing micro-hydropower generator,” Adv. Fluid Mech. 36, 73-84 (2003).

${ }^{7}$ B. J. Simpson, F. S. Hover, and M. S. Triantafyllou, "Experiments in direct energy extraction through flapping foils," in Proceedings of the Eighteenth International Offshore and Polar Engineering Conference, Canada, 6-11 July 2008.

${ }^{8}$ D. Huang and G. Wu, "Preliminary study on the aerodynamic characteristics of an adaptive reconfigurable airfoil," Aerosp. Sci. Technol. 27(1), 44-48 (2013).

${ }^{9}$ D. G. Huang, “A common unified computational fluid dynamic software and its validation," J. Eng. Thermophys. 33(10), 1699-1702 (2012).

${ }^{10}$ D. G. Huang, "A new preconditioning technology for fluid dynamics," J. Eng. Thermophys. 2(4), 593-595 (2005).

${ }^{11}$ L. P. Zhang, X. G. Deng, and H. X. Zhang, "Reviews of moving grid generation techniques and numerical methods for unsteady flow," Adv. Mech. 40(4), 424-447 (2010) (in Chinese).

${ }^{12} \mathrm{D}$. Li, Z. L. Lu, and T. Q. Guo, "An efficient dynamic mesh generation method for complex multi-block structured grid," Adv. Appl. Math. Mech. 6(1), 120-134 (2014).

${ }^{13}$ S. Yang, S. Luo, F. Liu, and H. Tsai, "Subsonic flow over unstalled pitching airfoil computed by Euler method," in Proceedings of the 36th AIAA Fluid Dynamics Conference and Exhibit, San Francisco, CA, USA, June 2006, pp. 2744-2759.

${ }^{14}$ T. Kinsey and G. Dumas, "Computational fluid dynamics analysis of a hydrokinetic turbine based on oscillating hydrofoil,” J. Fluids Eng. 134(2), 021104 (2012).

${ }^{15}$ B. J. Simpson, "Experimental studies of flapping foils for energy extraction," M.S. thesis, Massachusetts Institute of Technology, Cambridge, MA, USA, September 2009.

${ }^{16}$ Q. Zhu, "Optimal frequency for flow energy harvesting of a flapping foil," J. Fluid Mech. 675, 495-517 (2011).

${ }^{17} \mathrm{Q}$. Xiao, W. Liao, S. C. Yang, and Y. Peng, "How motion trajectory affects energy extraction performance of a biomimic energy generator with an oscillating foil?” Renewable Energy 37, 61-75 (2012).

${ }^{18}$ S. T. Davids, "A computational and experimental investigation of a flutter generator," M.S. thesis, Department of Aeronautics and Astronautics, Naval Postgraduate School, 1999. 
Journal of Renewable \& Sustainable Energy is copyrighted by AIP Publishing LLC (AIP). Reuse of AIP content is subject to the terms at: http://scitation.aip.org/termsconditions. For more information, see http://publishing.aip.org/authors/rights-and-permissions. 